\title{
How Far From Stability Can We Go Using Gammasphere and the ANL Fragment Mass Analyzer?
}

\author{
Michael P. Carpenter \\ Argonne National Laboratory \\ 9700 South Cass Ave. \\ Argonne, IL 60439
}

\begin{abstract}
Gammasphere is the pre-eminent detector for gamma-ray spectroscopy studies in the United States. The device consists of up to 110 Compton-Suppressed Ge detectors, and offers excellent energy resolution (2.3 keV at $1 \mathrm{MeV})$ and an order of magnitude increase in photopeak efficiency over previous Ge-arrays ( $10 \%$ at $1 \mathrm{MeV}$ ). Since early January 1998, Gammasphere has begun a cycle of experiments at the ATLAS accelerator at Argonne National Laboratory. As of this writing, 100 experiments have been carried out. A subset of these experiments have utilized Gammasphere coupled to the Fragment Mass Analyzer (FMA), a high resolution mass spectrometer which transports reaction products produced at the target position and disperses them by their mass/charge ratio at the focal plane. This presentation will highlight some of the physics issues being addressed by the utilization of these two devices in tandem. More specifically, experiments directed at studying (i) $\mathrm{N}=\mathrm{Z}$ nuclei at the edges of stability, (ii) the properties of excited states in proton emitters (iii) the stability and shapes of very heavy nuclei will be presented.
\end{abstract}

\section{INTRODUCTION}

The study of nuclei far from stability has received much attention recently. Indeed, the thrust of the next generation radioactive beam facilities will be to study nuclei which lie at or near the neutron drip line. On the other hand, nuclei which lie at the limits of proton excess have been produced and identified for elements as heavy as Bismuth $(Z=83)$. These isotopes were initially characterized by their decay properties, and little, if anything, was known about their excited states. A similar situation exists for the heaviest elements $(Z>100)$. Recently, with the coupling of Gammasphere [1] to other detection systems, it has become possible to identify $\gamma$ rays in nuclei lying at the proton drip line and in the heaviest elements. Consequently, studies of excited states in nuclei lying the furthest from stability have begun.

The submitted manuscript has been created by the University of Chicago as Operater of Argonne National Laboratory ("Argonne") under Contract No. W-31-109-ENG-38 with the U.S. Department of Energy. The U.S Government retains for itsell, and others act. ing on its behalf, a paid-up, nonexclusive irrevocable worldwide license in said article to reproduce, prepare derivative works, dis * tribute copies to the public, and perform pub. licly and display publicly, by or on behalf of the Government. 


\section{DISCLAIMER}

This report was prepared as an account of work sponsored by an agency of the United States Government. Neither the United States Government nor any agency thereof, nor any of their employees, make any warranty, express or implied, or assumes any legal liability or responsibility for the accuracy, completeness, or usefulness of any information, apparatus, product, or process disclosed, or represents that its use would not infringe privately owned rights. Reference herein to any specific commercial product, process, or service by trade name, trademark, manufacturer, or otherwise does not necessarily constitute or imply its endorsement, recommendation, or favoring by the United States Government or any agency thereof. The views and opinions of authors expressed herein do not necessarily state or reflect those of the United States Government or any agency thereof. 


\section{DISCLAIMER}

Portions of this document may be illegible in electronic image products. Images are produced from the best available original document. 


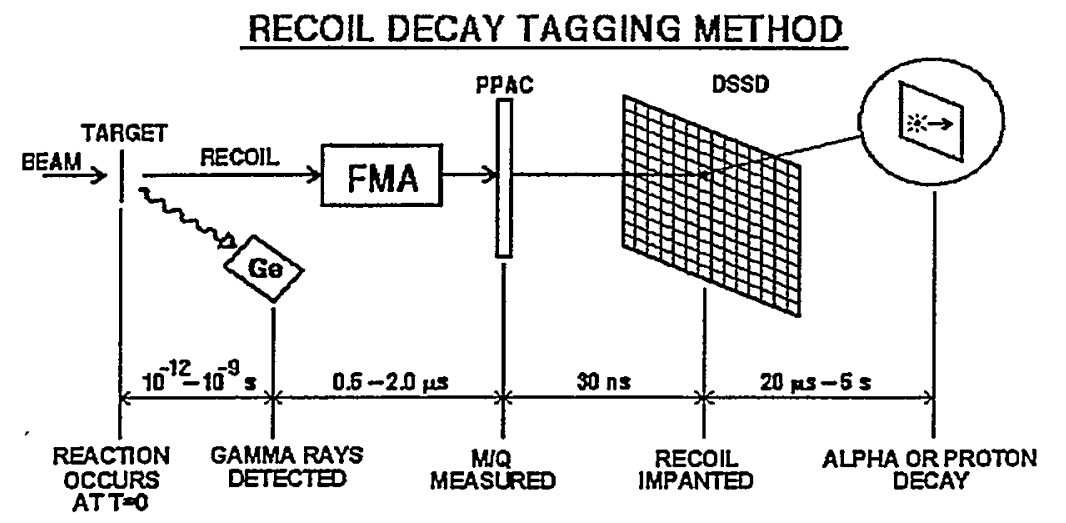

FIGURE 1. Schematic diagram of the Recoil Decay Tagging (RDT) Method as it is implemented at ATLAS. The method allows for clean isotopic identification of gamma rays detected at the target by correlating the implanted recoils with their characteristic charged-particle radioactivity.

Gammasphere is the pre-eminent detector for $\gamma$-ray spectroscopy studies in the world. The device consists of up to 110 Compton-Suppressed Ge detectors, and it was built by a collaboration of physicists from Argonne National Laboratory (ANL), Lawrence Berkeley National Laboratory (LBNL), Oak Ridge National Laboratory (ORNL), and a number of U.S. universities. The device offers excellent energy resolution $(2.3 \mathrm{keV}$ at $1 \mathrm{MeV})$ and an order of magnitude increase in photopeak efficiency over previous Ge-arrays ( $10 \%$ at $1 \mathrm{MeV})$. Between 1993-1997, the device was sited at the 88" Cyclotron Laboratory at LBNL where the major thrust of the experimental program was directed at the study of high-spin states in nuclei.

In the fall of 1997, Gammasphere was moved to the ATLAS accelerator at ANL and placed in front of the Fragment Mass Analyzer (FMA) in order to shift the emphasis of the experimental program from high-spin to nuclei far from stability. The FMA is a high resolution mass spectrometer which transports reaction products produced at the target position and disperses them by their mass/charge $(\mathrm{M} / \mathrm{Q})$ ratio at the focal plane, 8.8 meters away [2]. Presently, two general techniques are used at Gammasphere to make isotopic identification of weak channels produced in fusion-evaporation reactions. The first involves the measurement of evaporated particles, i.e. neutrons, protons, and/or alpha particles. The number and type of particles measured gives some degree of nuclide identification. The second technique directly detects the residue, and nuclide identification is made by measuring the properties of the residue, i.e. mass, energy, time of flight and/or decay products. Experiments with the FMA utilize this second technique.

While the FMA coupled to Gammasphere allows for mass identification of $\gamma$ rays, this is usually not sufficient for identifying the most proton-rich isotopes. This is due to the fact that after the fusion of the projectile and target, proton evaporation dominates over neutron evaporation in proton-rich compound systems. Since neutron evaporation leads to the production of systems lying furthest from stability, $\gamma$ rays emitted from nuclides with the largest proton excess become completely 


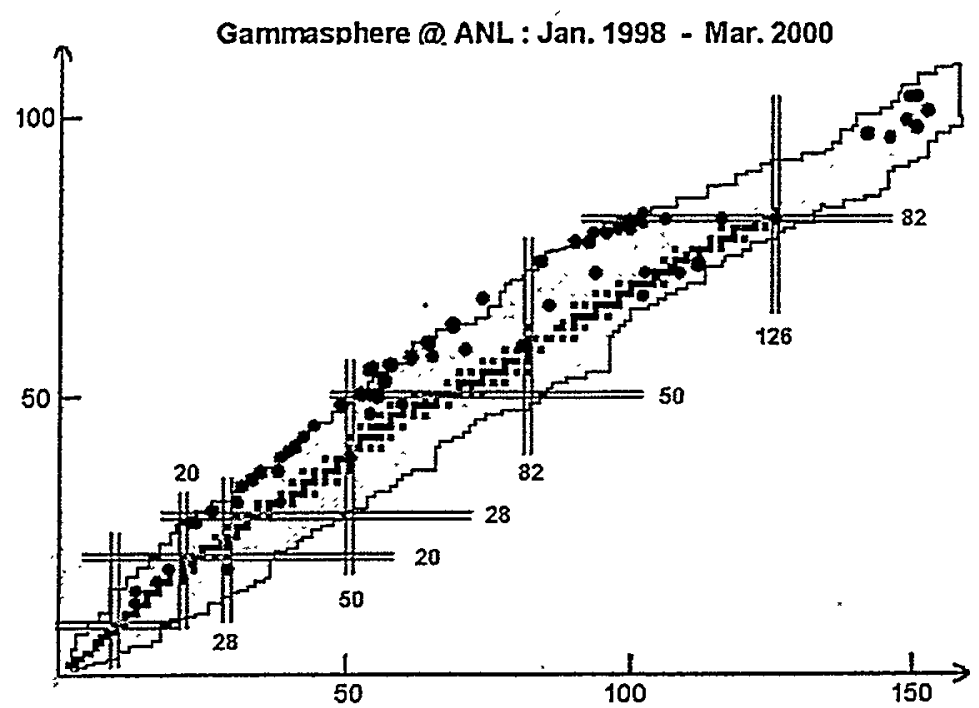

FIGURE 2. Known chart of the nuclides. Black squares represent stable nuclei. Circles represent nuclei which have been measured with Gammasphere at ATLAS between January, 1998 and March, 2000.

obscured by the $\gamma$ rays from the other isotopes produced in the heavy-ion reaction. Consequently, isotopic identification becomes necessary for isolating $\gamma$ transitions in these nuclei. With the FMA, this is achieved by placing ancillary detectors behind the focal plane. For light and medium mass nuclei $(\mathrm{Z}<50)$, it is possible to obtain isotopic selection by using an ion chamber. In heavier nuclei, isotopic identification of $\gamma$ rays is made by correlating the characteristic charged-particle radioactivity of an ion implanted in a pixel of a double-sided silicon strip detector (DSSD) with a previously implanted recoil. Figure 1 shows schematically how the technique works utilizing the DSSD setup at the FMA. This technique has wide applicability due to the fact that above the closed proton shell at $Z=50$, many nuclei near the proton-drip line decay by the emission of an alpha particle, and beyond the drip line, odd- $Z$ nuclides are observed to decay by proton emission. This technique is referred to as Recoil Decay Tagging (RDT) [3].

Experiments with Gammasphere coupled to the FMA were started in January, 1998 and will continue until March, 2000. Figure 2 gives a summary of the Gammasphere experiments performed at Argonne over this time. As the figure clearly illustrates, the majority of experiments has been directed at nuclei far from stability, i.e. along the proton-drip line or above $\mathrm{Z}=90$.

\section{STUDIES OF $\mathrm{N}=\mathrm{Z}$ NUCLEI}

The $N=Z$ nuclei between $Z=28$ and $Z=50$ closely follow the proton-drip line, and offer a particularly interesting laboratory for nuclear structure studies. In this 


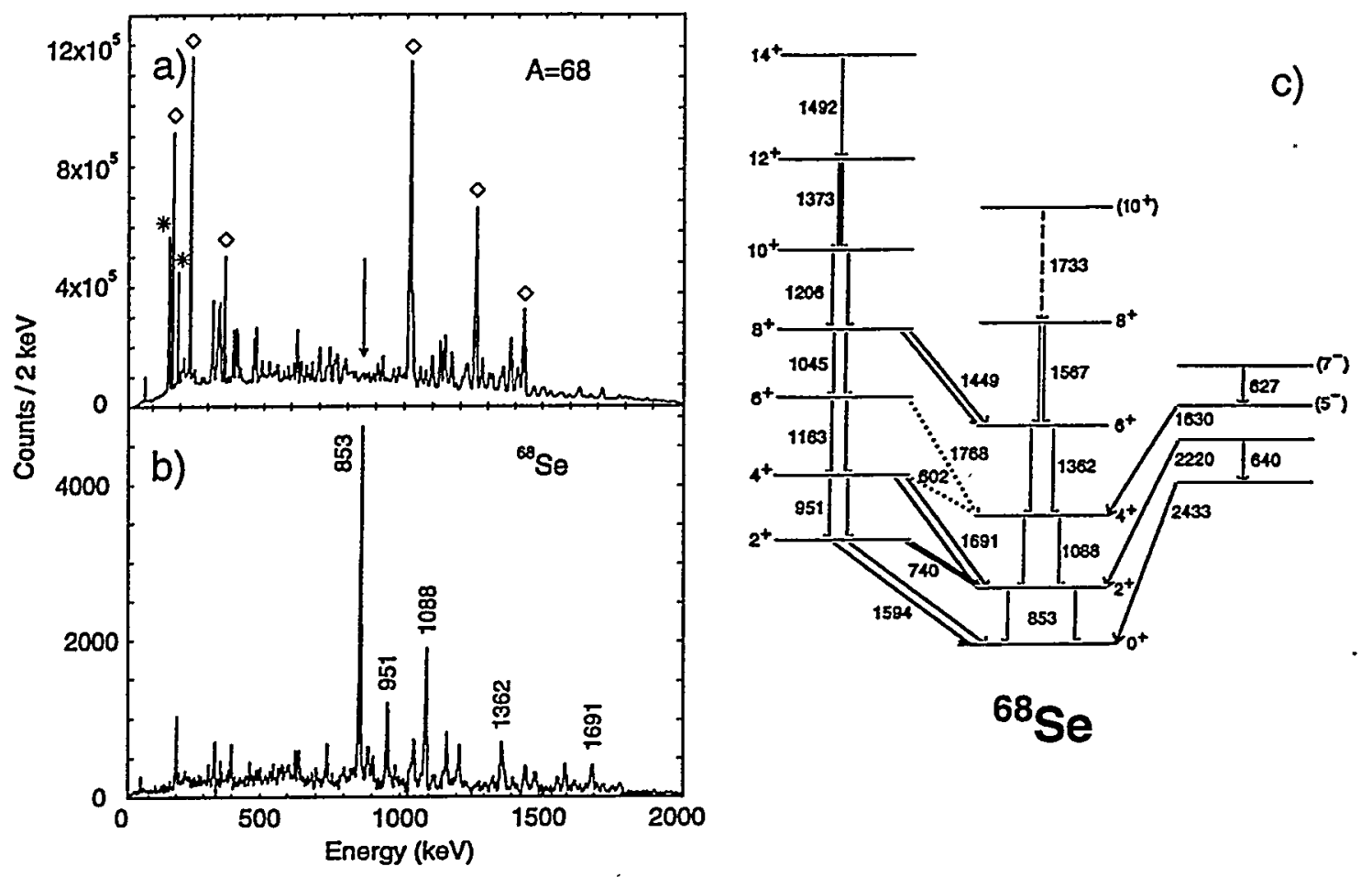

FIGURE 3. (a)Gamma-ray spectrum in coincidence with $A=68$ residues. (b) Gamma-ray spectrum associated with ${ }^{68} \mathrm{Se}$. (c) Deduced level structure for ${ }^{68} \mathrm{Se}$.

region, the single-particle spectrum shows large gaps as a function of deformation. The $\mathrm{N}=\mathrm{Z}$ symmetry reinforces these gaps, resulting in predictions of large shape changes and shape coexistence in these nuclei. Other issues of interest which are related to the $\mathrm{N}=\mathrm{Z}$ symmetry include measuring and quantifying the decline of isospin purity and determining the importance of $T=0$ and $1 \mathrm{n}-\mathrm{p}$ pairing.

As is evident in figure 2, a number of experiments directed at the study of $\mathrm{N}=\mathrm{Z}$ nuclei have been performed with Gammasphere. These measurements have fallen in two different classes. The first have utilized an array of CsI detectors, Microball, to measure evaporated charge particles [4] and an array of neutron counters which replace $\sim 30$ of the down-stream Ge detectors to measure evaporated neutrons. The second class of experiments have utilized the FMA and an ion chamber placed behind the FMA focal plane. By using highly inverse reactions, the energies of the residues lie above the Bragg curve. As a result, the mass of the recoil is determined by the FMA and $\mathrm{Z}$ identification of the residue is obtained from the $\Delta E$ signal measured in the ion chamber.

Recently, the $\mathrm{N}=\mathrm{Z}$ nucleus ${ }^{68} \mathrm{Se}$ has been measured at Gammasphere using the second technique. The ${ }^{12} C\left({ }^{58} \mathrm{Ni}, 2 \mathrm{n}\right){ }^{68} \mathrm{Se}$ reaction at 185 and $200 \mathrm{MeV}$ was utilized in this experiment to produce ${ }^{68} \mathrm{Se}$ with $\sim 250 \mu$ barn cross-section. Figure 3 demonstrates why the use of the ion chamber is necessary to identify. the ${ }^{68} \mathrm{Se}$ $\gamma$ rays. The upper part of the figure shows the spectrum of $\gamma$ rays in coincidence 
with $A=68$ residues, and it is dominated by ${ }^{68} \mathrm{Ge} \gamma$-rays. The bottom spectrum represents ${ }^{68} \mathrm{Se} \gamma$-rays after appropriate conditions are placed on the $\Delta E$ signal of the ion chamber. The ${ }^{68} \mathrm{Se} \gamma$ rays are indiscernible in the mass gated spectrum, and this highlights the need for isotopic identification in the study nuclei lying furthest from stability.

Figure 3 also shows the level structure for ${ }^{68} \mathrm{Se}$ deduced from the $\gamma$-ray coincidence data. Two rotational bands with strikingly different moments of inertia are observed. The moment of inertia deduced for the excited band is similar to that observed in neighboring nuclei and corresponds to a prolate shape with $\beta_{2} \sim+0.3$. In contrast, the moment of inertia of the ground-state band is smaller by $\sim 50 \%$ relative to the excited band and is consistent with the expectation for an oblate rotor with $\beta_{2} \sim-0.3$. The co-existence of both oblate and prolate structures in ${ }^{68} \mathrm{Se}$ supports the long-standing predictions for this nucleus. A more detailed description of these results can be found in ref. [5]

\section{STUDIES BEYOND THE PROTON DRIP-LINE}

Above $Z=50$, the limits of proton excess are defined by the proton emitters which have been identified in odd- $Z$ nuclei up to Bismuth $(Z=83)$. These nuclei lie beyond the proton drip line $\left(Q_{p} i \quad 0\right)$ and are kept bound by the Coulomb force. A number of new proton emitters have been identified recently using the FMA [6], and the lifetimes in nearly all of these cases can be well reproduced by WKB calculations using spectroscopic factors derived from spherical shell model calculations. This allows for definitive single-particle assignments to be given to the proton-emitting states. Recently, proton radioactivity has been observed in ${ }^{141} \mathrm{Ho}$ and ${ }^{131} \mathrm{Eu}$. However, the decay lifetimes cannot be reproduced by WKB calculations, and this has been interpreted as evidence for deformed grounds states in these nuclei [7]. By studying excited states built on top of these proton emitters, one should be able to independently confirm both the deformation and singleparticle parentage of the proton-emitting state. This is especially critical for the deformed emitters where there are only a few known cases. Other questions which can be addressed by studying excited states in proton emitters are:

- Do present models whose parameters have been adjusted using stable nuclei reproduce adequately nuclear structure all the way to the limits of stability, including deformations, single-particle energies, and the evolution of structure as a function of angular momentum?

- How much excitation energy and angular momentum can these quasi-bound systems accommodate?

- Is there any experimental evidence of mixing between bound and unbound states is these loosely bound systems?

Experimentally, such studies are difficult because the cross-sections for producing proton emitters are quite low $(<100 \mu \mathrm{barn})$. In addition, the $\gamma$-ray spectrum 

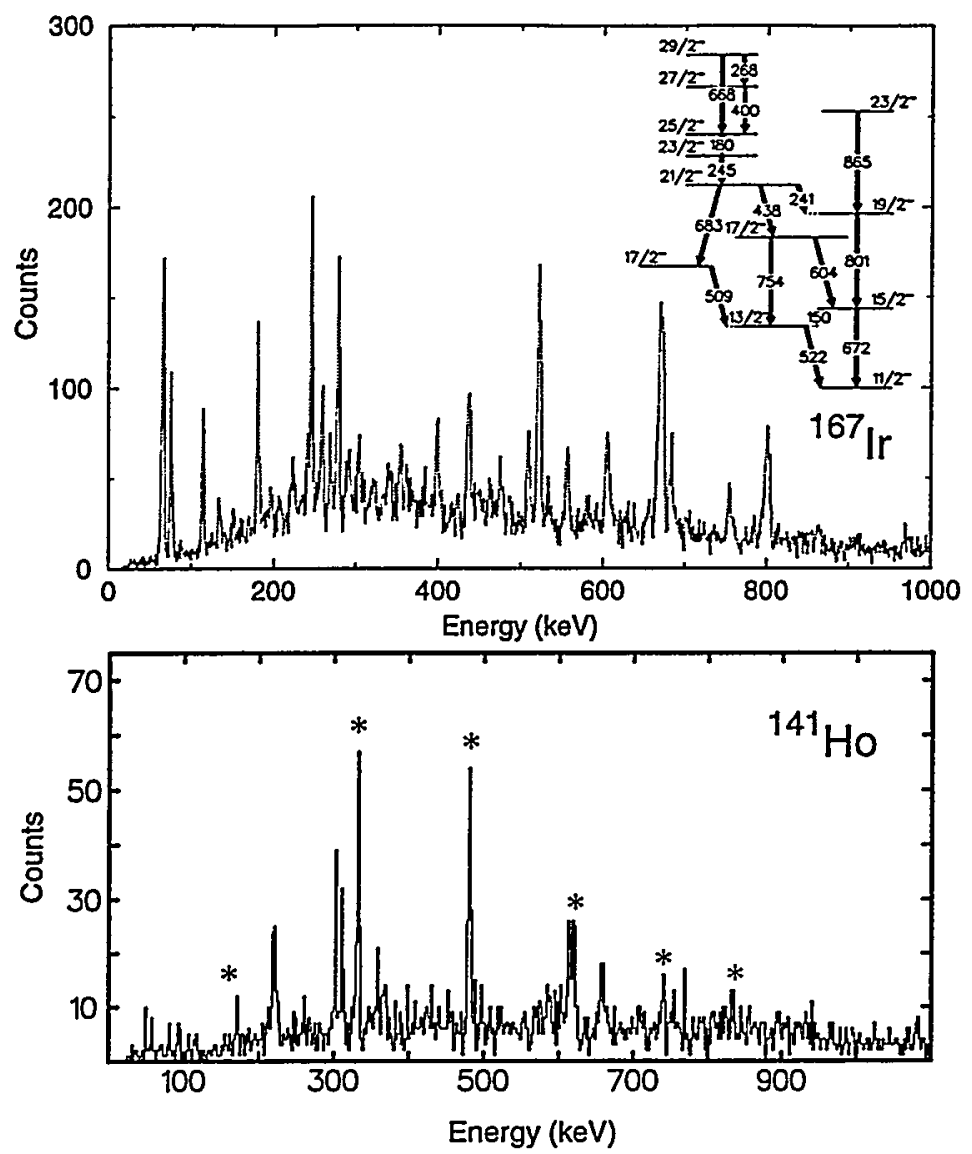

FIGURE 4. (Top) Gamma-ray spectrum correlated with the alpha/proton decay of the $h_{11 / 2}$ isomer state in ${ }^{167}$ Ir. The inset shows the partial level structure built on top of the isomer. (Bottom) Gamma-ray spectrum correlated with the ground-state proton decay of ${ }^{141} \mathrm{Ho}$. Transitions marked with "*" are members of the rotational band built on top of the ground state.

for an odd-A or odd-odd nucleus is in general more complicated than that of eveneven systems making $\gamma$-ray coincidence information necessary when determining the placement of even the first few excited states in a proton emitter. With the coupling of Gammasphere to the FMA, a 100 fold gain in $\gamma$ - $\gamma$-recoil efficiency has been realized over what was previously available, and this opens up the opportunity to perform $\gamma$-ray coincidence measurements on proton-emitters. Several such experiments have been performed, and two of them are described briefly below.

The two known alpha-emitting states in ${ }^{167} \mathrm{Ir}$ were recently found to proton decay as well [6]. Based on the partial half-lives, the ground state was given an $s_{1 / 2}$ assignment while the isomeric state at $175 \mathrm{keV}$ was assigned to the $\mathrm{h}_{11 / 2}$ configuration. The spectroscopic factors deduced for these states agree with the low-seniority shell model calculations which assume that all states are spherical. In order to study excited states in ${ }^{167} \mathrm{Ir}, \gamma$-recoil coincidences were measured with Gammasphere and the FMA using a $(\mathrm{p}, 2 \mathrm{n})$ reaction [8]. Figure 4 a shows the $\gamma$-ray spectrum correlated 
with the alpha decay of the isomeric level in ${ }^{167}$ Ir utilizing the RDT technique (fig. 1). These gamma rays are unresolvable in the mass-gated spectrum due to the fact that the latter is dominated by ${ }^{167} \mathrm{Os}(2 \mathrm{p}, \mathrm{n})$ and ${ }^{167} \mathrm{Re}(3 \mathrm{p})$ gamma rays. The inset shows the partial level scheme deduced from the $\gamma$-ray coincidence data (all spins are tentative). A sequence of gamma rays $(672,801,865 \mathrm{keV})$ is observed to feed the isomeric states. The pattern of excitation energy as a function of spin is consistent with a spherical or weakly deformed structure, and is thus, in agreement with the interpretation of the proton-decay data.

Recently, an experiment to measure excited states in ${ }^{141} \mathrm{Ho}$ was carried out with Gammasphere coupled to the FMA using the ${ }^{92} \mathrm{Mo}(54 \mathrm{Fe}, \mathrm{p} 4 \mathrm{n})$ reaction [9]. Despite the fact that the production cross section for ${ }^{141} \mathrm{Ho}$ is only $250 \mathrm{nb}, \gamma$ transitions in ${ }^{141} \mathrm{Ho}$ have been identified using the RDT technique. Fig. 4b shows the $\gamma$-ray spectrum correlated with ${ }^{141}$ Ho proton decays. Based on the $\gamma$-ray coincidence data, the transitions marked in the figure are found to be members of a rotational band. The deformation extracted from the band's moment of inertia agrees well with that deduced from the proton-decay half-life [7], but the absence of the expected signature partner band raises the possibility that a degree of triaxiality needs to be considered.

\section{STUDIES OF THE HEAVIEST ELEMENTS}

Identification of new elements is an ongoing activity in nuclear physics. Elements up to $\mathrm{Z}=118$ have been identified by measuring the energy of the alpha particle emitted when the nucleus decays. In many instances, the experimental quantities extracted from heavy-element decay studies, i.e. alpha-decay energies and alphadecay lifetimes, can only be reproduced by theoretical calculations by assuming that these nuclei are deformed. A more direct determination of the deformation can be obtained by measuring excited states in the nucleus of interest. For heavy nuclei, this is extremely difficult due to the fact that the production cross sections involved are at most a few $100 \mathrm{nb}$ and, typically, much less. There is one exception to this: the reaction using a ${ }^{48} \mathrm{Ca}$ beam to bombard a ${ }^{208} \mathrm{~Pb}$ target gives a maximum cross section of $3 \mu$ barn for producing ${ }^{254}$ No $(Z=102)$ via the $2 n$ channel.

Recently, a measurement was performed with Gammasphere in order to identify excited states in ${ }^{254}$ No using this reaction [10]. For this experiment, the FMA was essential to allow for the separation of ${ }^{254}$ No residues from the fission background produced at a $10^{4}$ higher rate. Unambiguous identification of ${ }^{254}$ No comes from the alpha-decay spectrum measured in the DSSD. The $\gamma$-ray spectrum obtained for ${ }^{254}$ No is shown in Fig. 5. Due to large conversion coefficients for low energy transitions, it is most likely that the level fed by the $159 \mathrm{keV}$ transition is not the ground state. In order to estimate the spins of the levels connected by the observed transitions and the energies of the missing transitions, the observed $\gamma$ rays have been fit using a rotational model. Following this prescription, it was deduced that the $159 \mathrm{keV}$ transition feeds the $6^{+}$level, and the two missing transitions have 


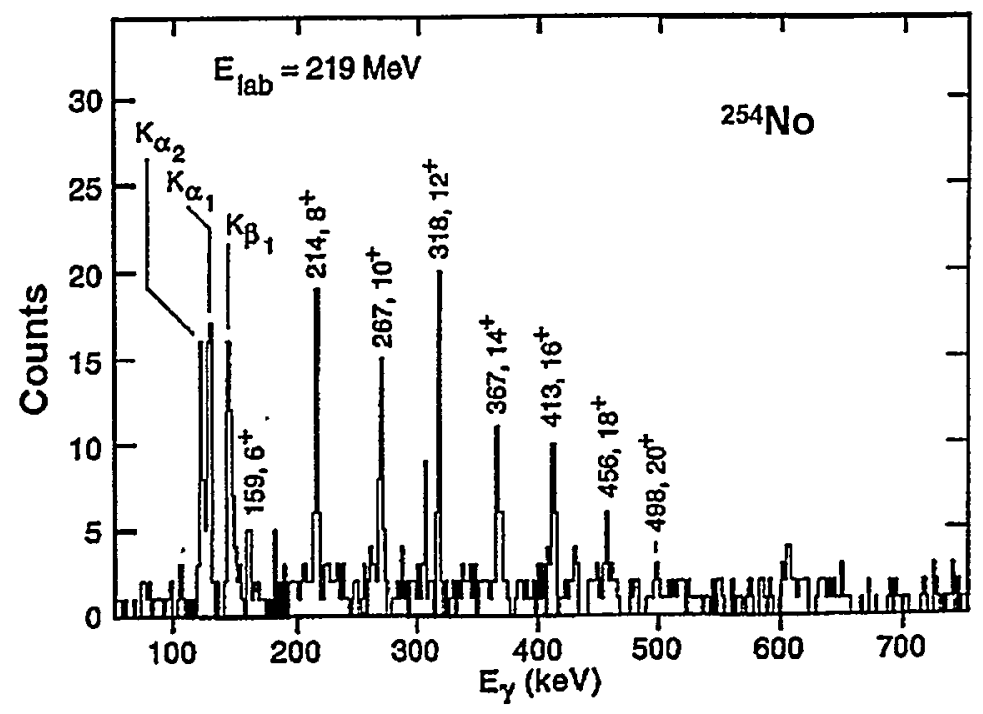

FIGURE 5. Recoil gated Gamma-ray spectrum for ${ }^{254}$ No.

energies of 44 and $102 \mathrm{keV}$. The last identified gamma-ray would then correspond to the $20^{+}$to $18^{+}$transition in ${ }^{254}$ No. In addition, the quadrupole deformation is estimated at 0.27 which agrees well with theoretical predictions. It should also be noted that similar results have been reported in ref. [11].

\section{SUMMARY}

Gammasphere has operated in tandem with the ANL Fragment Mass Analyzer for two years. In this time, a wealth of new information has been collected with regards to excited states in nuclei at the proton drip-line as was well as in heavy elements. In this presentation, several examples have been given which illustrate the power of the two devices when coupled together. I would like to thank my ANL colleagues who have worked with me these past two years to operate Gammasphere at Argonne. This work is supported by the U.S. Dept. of Energy under contract No. W-31-109-ENG-38.

\section{REFERENCES}

1. I.Y. Lee, Nucl. Phys. A520, 641 (1990).

2. C.N. Davids et al., Nucl. Inst. Meth. Phys. Res. B70, 358 (1992).

3. E.S. Paul et al., Phys. Rev. C51, 78 (1995).

4. D.G. Sarantites et al., Nucl. Instr. and Meth. A381, 418 (1996). 
5. S.M. Fischer et al., accepted for publication in Phys. Rev. Letters.

6. C.N. Davids et al., Phys. Rev. C55, 2255 (1997).

7. C.N. Davids et al., Phys. Rev. Lett. 80, 1849 (1998).

8. M.P. Carpenter et al., Acta Phys. Pol. B30, 551 (1999).

9. D. Seweryniak et al., to be published.

10. P. Reiter et al., Phys. Rev. Lett. 82, 509 (1999) and P. Reiter et al., accepted for publication in Phys. Rev. Letters.

11. M. Leino et al., Euro. Phys. Jour. A6, 63 (1999). 